\title{
Analysis of ureteral length in adult cadavers
}

\author{
Hugo F. F. Novaes, Paula C. S. Leite, Rafaela A. Almeida, Ney C. B. Sorte, Ubirajara Barroso Júnior \\ Urology Department, Fundação para o Desenvolvimento da Ciência. Escola Bahiana de Medicina e Saúde \\ Pública, Bahia, Brazil
}

\section{ABSTRACT}

Introduction: In some occasions, correlations between human structures can help planning surgical intra-abdominal interventions. The previous determination of ureteral length helps pre-operatory planning of surgeries, reduces costs of auxiliary exams, the correct choice of double-J catheter with low morbidity and fewer symptoms, and an adequate adhesion to treatment.

Objective: To evaluate ureteral length in adult cadavers and to analyze its correlation with anthropometric measures.

Materials and Methods: From April 2009 to January 2012 we determined ureteral length of adult cadavers submitted to necropsy and obtained the following measures: height, distance from shoulder to wrist, elbow-wrist, xiphoid appendix-umbilicus, umbilicus-pubis, xiphoid appendix-pubis and between iliac spines. We analyzed the correlations between ureteral length and those anthropometric measures.

Results: We dissected 115 ureters from 115 adult corpses from April 2009 to January 2012. Median ureteral length didn't vary between sexes or according to height. It was observed no correlation among ureteral length and all considered anthropometric measures in all analyzed subgroups and in general population. There were no significant differences between right and left ureteral measures.

Conclusions: There is no difference of ureteral length in relation to height or gender (male or female). There is no significant correlation among ureteral length and the considered anthropometric measures.

\section{ARTICLE INFO}

\section{Key words:}

Ureter; Body Height; Stents;

Human Engineering; Cadaver

Int Braz J Urol. 2013; 39: 248-56

Submitted for publication:

August 22, 2012

Accepted after revision:

December 21, 2012

\section{INTRODUCTION}

The search of human body proportionality is ancient and has not ended. The first recorded observation of human body proportions was made by the roman architect Vitruvius, posteriorly disseminated by Leonardo da Vinci's masterpiece "The Vitruvian man" (1).

In some occasions, correlations between different human structures could help planning intra-abdominal surgeries. In the era of minimally invasive surgeries, the previous knowledge of ureteral length allows a correct planning of a reconstructive surgery or ureteral reimplantation, redu- cing costs, without the need of invasive subsidiary exams, such as cystoscopy or radiological profiles (for example, intravenous pyelography or computerized tomography). The correct choice of the length of a double-J catheter reduces symptoms and morbidity (2-7) and one of the determinants is the catheter length $(3,8,9)$. A very long catheter can cause irritative symptoms, while a short one allows migration $(3,8,10,11)$.

Some authors searched for correlations between ureteral length and anthropomorphic measures $(2,3,10,12)$ and some have found poor 
correlations with no clinical use $(2,12)$, while others haven't found any $(3,10)$. Such studies were conducted using image or endoscopic methods.

There are no Brazilian data about ureteral length or studies about correlations between ureteral and anthropometric measures using cadavers. So we decided to dissect ureters of human cadavers submitted to necropsies at the medical legal institute, and determined their lengths and correlations with some anthropometric measures. According to our knowledge, this is the first Brazilian study that correlates ureteral length with anthropometric data and the first in medical literature to determine these measures in cadavers (the measurement is more accurate than other methods being used so far).

\section{MATERIALS AND METHODS}

This is a transverse study that evaluates the length of ureters (point of interest) and other anthropometric measures (predictors) in one particular moment. Data were collected from April 2009 to January 2012 and were obtained from the dissection of cadaveric ureters of patients with more than 16 years old, submitted to necropsy at the Medical Legal Institute Nina Rodrigues, in Salvador, Bahia.

The necropsied cadavers (with violent or natural cause of death) were submitted to retroperitoneal dissection (Figure-1), separating and removing the kidney and corresponding ureter with its bladder insertion (Figure-2). Renal pelvis

\section{Figure 1 - Retroperitoneum.}

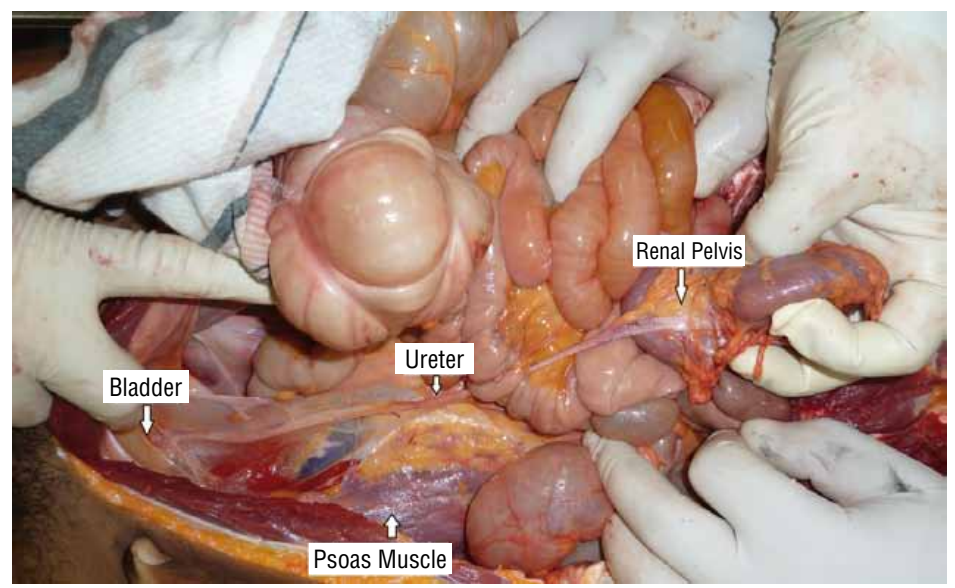

Figure 2 - Kidney, ureter and bladder cuff.

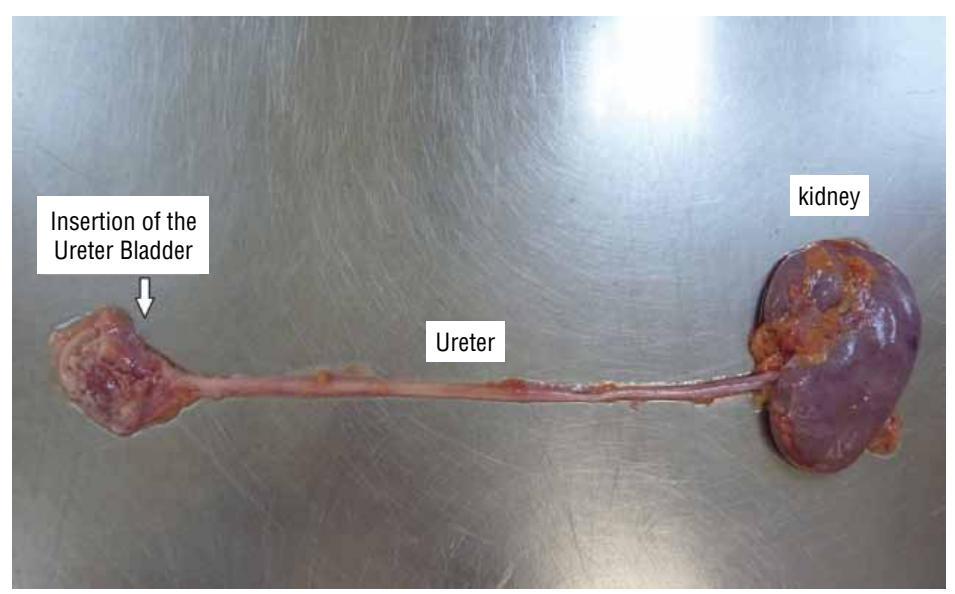


was detached from the renal parenchyma and the whole structure (renal pelvis, ureter and bladder insertion) was rectified at the bench (Figure-3). The ureter was delimited by the ureteral-pielic junction (UPJ) and ureteral-bladder junction (UBV). UPJ was identified at the point of proximal enlargement of the ureter and the UBV at the point of distal enlargement. In case of doubt, the extremities were opened longitudinally to help identify these points (Figure-4). The dissection was done bilaterally and at the bench the structure was rectified in order to measure the ureteral length using a millimetric scale with $5 \mathrm{~mm}$ gradation.

The following measures were also obtained: distance from shoulder to wrist (acromial process-head of the ulna), elbow-wrist (olecranon-head of the ulna), xiphoid appendix-umbilicus, umbilicus-pubis, xiphoid appendix-pubis and between iliac spines. The cadavers were randomly chosen, and those with primary or secondary ureteral diseases leading to anatomic distortion (megaureter, neoplasms, adhesions, duplicity), ureteral trauma or previous ureteral surgery (confirmed or suspected) or any evident ureteral disease were excluded.

Calculation of the sample was made using the software BioEstat 5.0, based on the data of Jeon et al. (4), that described a correlation between height and ureteral length with a correlation coefficient $r=0.369$. With a statistical power of

Figure 3 - Ureter (red arrows indicate UPJ at left and UBJ at right).

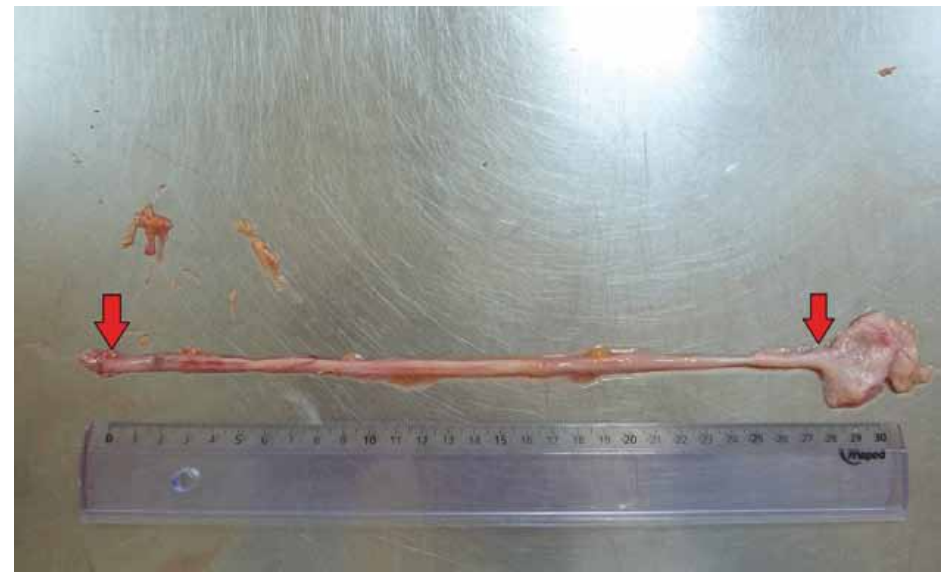

Figure 4 - UPJ after longitudinal incision.

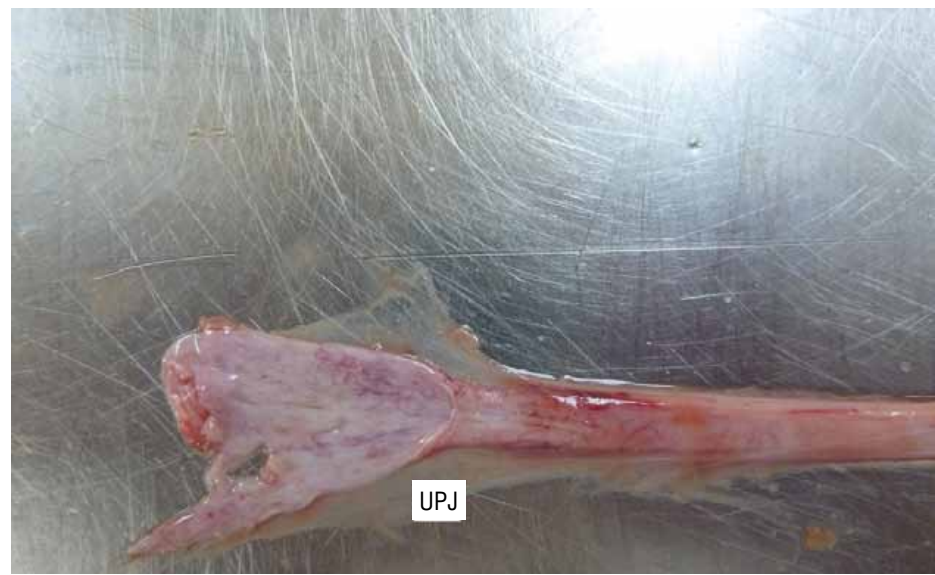


$90 \%$ with a type error I of 5\%, it was verified the need of a sample of 83 ureters.

For statistical analysis, the sample was categorized according to gender (male and female) and height (subgroup H1: cadavers with less than 165 $\mathrm{cm}$ height, subgroup H2: height from 166 to 175 $\mathrm{cm}$; and subgroup H3: height equal or superior to $176 \mathrm{~cm}$ ). Student t test was used for the analysis of the ureteral length according to gender. For analysis of ureteral length according to height subgroups, it was used analysis of variance (ANOVA).

Pearson correlation was used to analyze the correlation between ureteral length and the anthropometric measures. Multiple linear regression was used to evaluate the possibility of obtaining a predictor equation of ureteral length. Backward strategy was used to determine the predictive variables of the final model. In order to correct the possible effects of extreme values, it was obtained correlation and regression analysis of all values of ureteral lengths and to all values that, in a normal standardized scale, had z scores of -1.96 to 1.96 .

$\mathrm{P}$ values lower than $5 \%(\mathrm{p}<0.05)$ were considered significant. All analysis were made using SPSS software version 14.0.

This study was approved by the Medical Ethical Committee of our institution and by the scientific board of the medical legal institute.

\section{RESULTS}

The ureters of 115 adult cadavers were dissected, with ages equal or superior to 16 years old, from April 2009 to January 2012. Twenty two were female and 93 male (1 female: 4.22 male). Median female age (standard deviation) was 51.48 $( \pm 13.63)$ and $38.95( \pm 19.0)$ male $(p=0.002)$. Median (standard deviation) height was 159.5 ( \pm 6.36) for females and $171.59( \pm 7.42)$ for males $(p$ $\leq$ 0.001) (Table-1).

Table 1 - Different aspects of the studied population.

\begin{tabular}{lccc}
\hline & $\begin{array}{c}\text { Female } \\
\text { Median }(\mathrm{sd})\end{array}$ & $\begin{array}{c}\text { Male } \\
\text { Median }(\mathrm{sd})\end{array}$ & $\mathrm{P}$ \\
\hline Age & $51.48( \pm 13.63)$ & $38.95( \pm 18.99)$ & $<0.001$ \\
Height & $159.50( \pm 6.36)$ & $171.59( \pm 7.42)$ & $<0.001$ \\
SW distance & $52.68( \pm 2.76)$ & $57.57( \pm 4.21)$ & $<0.001$ \\
SE distance & $28.47( \pm 2.04)$ & $31.07( \pm 3.28)$ & $<0.001$ \\
EW distance & $24.20( \pm 1.68)$ & $26.49( \pm 1.76)$ & 0.546 \\
XU distance & $19.40( \pm 3.09)$ & $20.02( \pm 3.45)$ & 0.635 \\
UP distance & $16.63( \pm 2.28)$ & $17.05( \pm 2.39)$ & 0.618 \\
XP distance & $36.04( \pm 3.45)$ & $36.38( \pm 5.01)$ & 0.004 \\
IS-IS & $25.45( \pm 2.97)$ & $23.30( \pm 2.62)$ & 0.135 \\
Ureteral length & $26.73( \pm 4.84)$ & $25.05( \pm 3.27)$ &
\end{tabular}

SW: Shoulder-wrist; SE: Shoulder-elbow; EW: Elbow-wrist; XU: xiphoid appendix-umbilicus; UP: umbilicus-pubis; XP: xiphoid appendix-pubis; IS-IS: between antero-superior iliac spines. 
Medial ureteral length (standard deviation) was $25.36( \pm 3.67) \mathrm{cm}$. When we analyzed ureteral length according to gender, there was no significant differences ( $p=0.135)$, although female ureters presented a median length (sd) of 26.73 ( \pm 4.85) and male ureters $25.05( \pm 3.26) \mathrm{cm}$ (Table-1).

When we analyzed the height subgroups, there were also no statistical differences of ureteral length: medial length (sd) of subgroups $\mathrm{H} 1, \mathrm{H} 2$ and H3 were respectively $25.09( \pm 4.88) \mathrm{cm}, 25.51$ $( \pm 2.99) \mathrm{cm}$ and $25.46( \pm 3.07) \mathrm{cm}(\mathrm{p}=0.860)$.

Among 115 analyzed cadavers, there were only 7 with different contralateral measures of ureters, varying from 1.5 to $6.5 \mathrm{~cm}$. In these cases, left ureter was longer than the contralateral in three corpses. No pathologies were identified in these 7 cadavers.

There were no correlations between ureteral length and anthropometric measures in general population (Table-2). When the sample was categorized according to gender, there was also no correlation (Table-2). There were low correlations in some analysis of the population categorized according to height. Among the 34 cadavers of subgroup H1, ureteral length correlated with the shoulder-elbow distance, with a Pearson index of -0.368 ( $p=0.038)$. In subgroup $\mathrm{H} 2$, there was also a low correlation between height and ureteral length (Pearson index -0.337 and $p=0.013$ ). In subgroup H3, it was identified a correlation between height and ureteral left length, with Pearson index of -0.292 and $p=0.042$ (Table-3). And finally, when we categorized the population simultaneously according to gender and height, there were no significant correlations (Tables 4 and 5).

\section{DISCUSSION}

Median ureteral length was 14 to $37 \mathrm{~cm}$ (median $25.36 \pm 3.67 \mathrm{~cm}$ ) in accordance to literature $(16$ to $35 \mathrm{~cm})(2-4,8,10)$. There was no statistical significant difference between men $(25.05 \pm$ $3.27 \mathrm{~cm})$ and women $(26.73 \pm 4.84 \mathrm{~cm})(\mathrm{p}=0.135)$, although median height of male population was bigger $(171.59 \pm 7.42 \mathrm{~cm}$ male and $159.50 \pm 6.36$ $\mathrm{cm}$ female) $(\mathrm{p}<0.001)$. The cause of this finding is unknown, but probably due to the larger pelvis of women, with a longer ureteral path. There

Table 2 - Correlations between ureteral length and several anthropometric measures.

\begin{tabular}{lccc}
\hline & $\begin{array}{c}\text { General population } \\
\text { Pearson (p value) }\end{array}$ & $\begin{array}{c}\text { Male } \\
\text { Pearson (p value) }\end{array}$ & $\begin{array}{c}\text { Female } \\
\text { Pearson (p value) }\end{array}$ \\
\hline Height & $-0.012(0.900)$ & $0.124(0.244)$ & $0.090(0.689)$ \\
Shoulder-wrist distance & $-0.098(0.310)$ & $0.002(0.982)$ & $-0.164(0.466)$ \\
Shoulder-elbow distance & $-0.118(0.221)$ & $-0.029(0.786)$ & $-0.266(0.231)$ \\
Elbow-wrist distance & $-0.025(0.794)$ & $0.061(0.575)$ & $0.054(0.812)$ \\
Xiphoid appendix-umbilicus & $-0.079(0.414)$ & $-0.078(0.470)$ & $-0.049(0.827)$ \\
distance & & & $-0.157(0.484)$ \\
Umbilicus-pubis distance & $0.111(0.249)$ & $-0.084(0.438)$ & $-0.149(0.509)$ \\
$\begin{array}{l}\text { Xiphoid appendix-pubis } \\
\text { distance }\end{array}$ & $-0.113(0.241)$ & $-0.109(0.317)$ & \\
Iliac spines distance & $0.172(0.073)$ & $0.111(0.303)$ & $0.180(0.423)$ \\
\hline
\end{tabular}


Table 3 - Correlations between ureteral length and other anthropometric measures in general population according to height.

\begin{tabular}{lccc}
\hline & $\begin{array}{c}\mathrm{H} 1 \\
\text { Pearson ( } \mathrm{p} \text { value })\end{array}$ & $\begin{array}{c}\mathrm{H} 2 \\
\text { Pearson ( } \mathrm{p} \text { value })\end{array}$ & $\begin{array}{c}\mathrm{H} 3 \\
\text { Pearson ( } \mathrm{p} \text { value })\end{array}$ \\
\hline Height & $-0.119(0.496)$ & $-0.337(0.013)$ & $0.329(0.116)$ \\
Shoulder-wrist distance & $-0.316(0.073)$ & $-0.058(0.683)$ & $0.127(0.562)$ \\
Shoulder-elbow distance & $-0.363(0.038)$ & $-0.060(0.672)$ & $0.117(0.596)$ \\
Elbow-wrist distance & $-0.137(0.447)$ & $-0.012(0.931)$ & $0.119(0.588)$ \\
Xiphoid appendix-umbilicus distance & $-0.096(0.594)$ & $-0.237(0.090)$ & $0.118(0.593)$ \\
Umbilicus-pubis distance & $-0.096(0.595)$ & $0.034(0.811)$ & $-0.429(0.046)$ \\
Xiphoid appendix-pubis distance & $-0.144(0.424)$ & $-0.127(0.369)$ & $0.053(0.814)$ \\
Iliac spines distance & $0.249(0.163)$ & $0.088(0.537)$ & $0.178(0.418)$ \\
\hline
\end{tabular}

Obs: The indicated values in the table refer to Pearson coefficients and the significance of parametrical analysis (). H1: Cadavers with height equal or inferior to $165 \mathrm{~cm}$; H2: cadavers with height from 166 to $175 \mathrm{~cm}$; H3: cadavers with height equal or superior to $176 \mathrm{~cm}$.

Table 4 - Correlations between ureteral length and other anthropometric measures in male population according to height.

\begin{tabular}{llll}
\hline & $\begin{array}{c}\mathrm{H} 1 \\
\text { Pearson ( } \mathrm{p} \text { value })\end{array}$ & $\begin{array}{c}\mathrm{H} 2 \\
\text { Pearson (p value) }\end{array}$ & $\begin{array}{c}\mathrm{H} 3 \\
\text { Pearson (p value) }\end{array}$ \\
\hline Height & $-0.124(0.625)$ & $-0.322(0.024)$ & $0.329(0.116)$ \\
Shoulder-wrist distance & $-0.258(0.335)$ & $-0.041(0.783)$ & $0.127(0.562)$ \\
Shoulder-elbow distance & $-0.162(0.548)$ & $-0.059(0.693)$ & $0.117(0.596)$ \\
Elbow-wrist distance & $-0.334(0.207)$ & $-0.030(0.840)$ & $0.119(0.588)$ \\
Xiphoid appendix-umbilicus distance & $-0.295(0.267)$ & $-0.268(0.069)$ & $0.118(0.593)$ \\
Umbilicus-pubis distance & $-0.089(0.744)$ & $0.029(0.847)$ & $-0.429(0.046)$ \\
Xiphoid appendix-pubis distance & $-0.108(0.690)$ & $-0.154(0.303)$ & $0.053(0.814)$ \\
Iliac spines distance & $0.227(0.398)$ & $0.002(0.987)$ & $0.178(0.418)$ \\
\hline
\end{tabular}

Obs: The indicated values in the table refer to Pearson coefficients and the significance of parametrical analysis (). H1: Cadavers with height equal or inferior to $165 \mathrm{~cm}$; H2: cadavers with height from 166 to $175 \mathrm{~cm}$; H3: cadavers with height equal or superior to $176 \mathrm{~cm}$. 
Table 5 - Correlations between ureteral length and other anthropometric measures in the female population according to height.

\begin{tabular}{llc}
\hline & $\begin{array}{c}H 1 \\
\text { Pearson (p value) }\end{array}$ & P2 \\
\hline Height & $-0.017(0.948)$ & $-0.526(0.362)$ \\
Shoulder-wrist distance & $-0.273(0.288)$ & $0.103(0.870)$ \\
Shoulder-elbow distance & $-0.477(0.053)$ & $0.158(0.800)$ \\
Elbow-wrist distance & $-0.019(0.942)$ & $-0.395(0.511)$ \\
Xiphoid appendix-umbilicus distance & $-0.011(0.966)$ & $0.229(0.710)$ \\
Umbilicus-pubis distance & $-0.145(0.578)$ & $-0.158(0.800)$ \\
Xiphoid appendix-pubis distance & $-0.121(0.643)$ & $0.103(0.870)$ \\
Iliac spines distance & $0.112(0.668)$ & $0.289(0.637)$
\end{tabular}

Obs: The indicated values in the table refer to Pearson coefficients and the significance of parametrical analysis (). H1: Cadavers with height equal or inferior to $165 \mathrm{~cm}$; H2: cadavers with height from 166 to $175 \mathrm{~cm}$; H3: cadavers with height equal or superior to $176 \mathrm{~cm}$.

was also no statistical difference between distances in both sexes (xiphoid appendix-pubis ( $\mathrm{p}$ $=0.618)$, xiphoid appendix-umbilicus $(p=0.546)$ and umbilicus-pubis ( $p=0.635$ ) independently of the subgroups of height, suggesting that the abdominal length of ureter doesn't alter according to gender or subgroup of height, and therefore, the distance between the kidney and the bladder may also not vary.

There was also no statistical difference of ureteral length according to the subgroups of height. Median ureteral length in subgroup H1 (height equal or lower than $165 \mathrm{~cm}$ ) was $25.09 \pm 4.88$ $\mathrm{cm}$, in subgroup H2 (height from 166 to $175 \mathrm{~cm}$ ) $25.51 \pm 2.99 \mathrm{~cm}$ and in subgroup H3 (height equal or superior to $176 \mathrm{~cm}$ ) was $25.46 \pm 3.07 \mathrm{~cm} \mathrm{(p=}$ 0.860). Accordingly, we observed that correlation between height and abdominal length (represented by the distances from xiphoid appendix-umbilicus, umbilicus-pubis, xiphoid appendix-pubis) is low $(\mathrm{r}=0.282$ with $\mathrm{p}=0.003 ; \mathrm{r}=0.295$ with $\mathrm{p}=$ 0.002 ; and $r=0.227$ with $p=0.019$, respectively), disqualifying the affirmative that higher people have longer ureters. So, the choice of a double-J catheter should not be based upon height and is recommended the use of a median length catheter to all population.

In only 7 of a total of 115 necropsied cadavers there were differences between length of right and left ureters. Hruby et al. (2) analyzed 100 patients with endoscopy and also didn't find any difference, while Paick et al. (3) found $1 \mathrm{~cm}$ difference between left and right ureters in a sample of 203 patients. The $5 \mathrm{~mm}$ scale used in our work could justify the lack of observance of small differences between contralateral units. Another difficulty to compare data with living beings is related to the way of obtaining the measures. In vivo, ureters can contract or dilate according to manipulation, and image exams may not reflect the correct measure of the ureteral length.

There was no significant correlation between ureteral length and the studied anthropometric measures, in general population or according to gender or height. Hruby et al. (2) in a similar study used endoscopy and radioscopy to measure ureteral length and found a weak correlation with height, xiphoid appendix-pubis distance and 
elbow-wrist distance, and established an equation that predicts ureteral length in only 26\% (2). Breau et al. (12) and Jeon et al. (4) also reported weak correlations between height and ureteral length. Shah et al. (10) and Pilcher et al. (8) found no correlations between height and ureteral length. In view of these facts, this study reaffirms previous works regarding the lack of correlation between different segments of the human body and the ureteral length, making impossible the proposal of a predictive formula. The absence of correlation between height and ureteral length would also explain the absence of difference of ureteral length according to different heights.

Among all limitations of this study, we point out that the sample was a convenient one, that represented the black population with low income of Salvador (except for one white cadaver, all others were black or brown). Brazil has a vast territorial extension, with a very heterogeneous population with African, European and Asiatic descendents. And these differences are more evident in different regions of the country, with different median heights. Low income populations are more prone to deficits of physical development, with lower heights. However, since it was not found any correlation between height and all other anthropometric measures, probably this bias did not interfere the results.

\section{CONCLUSIONS}

There are no significant statistical differences in median ureteral length according to different genders or heights; median length is 25.36 $( \pm 3.67) \mathrm{cm}$.

There is not a significant correlation between ureteral length and all evaluated segments of human body, making impossible the prediction of the length of the ureter accordingly.

\section{CONFLICT OF INTEREST}

None declared.

\section{REFERENCES}

1. Schott GD: The extent of man from Vitruvius to Marfan. Lancet. 1992; 340: 1518-20.

2. Hruby GW, Ames CD, Yan Y, Monga M, Landman J: Correlation of ureteric length with anthropometric variables of surface body habitus. BJU Int. 2007; 99: 1119-22.

3. Paick SH, Park HK, Byun SS, Oh SJ, Kim HH: Direct ureteric length measurement from intravenous pyelography: does height represent ureteric length? Urol Res. 2005; 33: 199-202.

4. Jeon SS, Choi YS, Hong JH: Determination of ideal stent length for endourologic surgery. J Endourol. 2007; 21: 906-10.

5. Ho CH, Huang KH, Chen SC, Pu YS, Liu SP, Yu HJ: Choosing the ideal length of a double-pigtail ureteral stent according to body height: study based on a Chinese population. Urol Int. 2009; 83: 70-4.

6. Lee BK, Paick SH, Park HK, Kim HG, Lho YS: Is a $22 \mathrm{~cm}$ Ureteric Stent Appropriate for Korean Patients Smaller than $175 \mathrm{~cm}$ in Height? Korean J Urol. 2010; 51: 642-6.

7. Kawahara $\mathrm{T}$, Ito $\mathrm{H}$, Terao $\mathrm{H}$, Yoshida M, Ogawa $\mathrm{T}$, Uemura $\mathrm{H}$, et al.: Choosing an appropriate length of loop type ureteral stent using direct ureteral length measurement. Urol Int. 2012; 88: 48-53.

8. Pilcher JM, Patel U: Choosing the correct length of ureteric stent: a formula based on the patient's height compared with direct ureteric measurement. Clin Radiol. 2002; 57: 59-62.

9. $\mathrm{Ho} \mathrm{CH}$, Chen SC, Chung SD, Lee YJ, Chen J, Yu HJ, et al.: Determining the appropriate length of a double-pigtail ureteral stent by both stent configurations and related symptoms. J Endourol. 2008; 22: 1427-31.

10. Shah J, Kulkarni RP: Height does not predict ureteric length. Clin Radiol. 2005; 60: 812-4.

11. Slaton JW, Kropp KA: Proximal ureteral stent migration: an avoidable complication? J Urol. 1996; 155: 58-61.

12. Breau RH, Norman RW: Optimal prevention and management of proximal ureteral stent migration and remigration. J Urol. 2001; 166: 890-3.

Correspondence address: Dr. Hugo F. F. Novaes Urology Department, Fundação para o Desenvolvimento da Ciência Escola Bahiana de Medicina e Saúde Pública, Bahia, Brazil Rua Clara Nunes, 543 / 702, Pituba Salvador, Bahia, 41810-425, Brazil E-mail: hugonovaesbr@yahoo.com.br 


\section{EDITORIAL COMIMENT}

The fact that few studies on the anatomy ureteral in the years fifty and seventy to reflect the originality of this article highlighted $(1,2)$. As for its clinical applicability in adult surgical matter of finding not occur significant difference in mean length ureteral between different age and height between males and females, and the average length of $25.36( \pm 3.67) \mathrm{cm}$. As the author rightly emphasizes the difficulty of com- paring the data in the literature relates to the way of obtaining measurements, since in vivo, the ureter can contract and shorten as their handling, and imaging studies may not reflect the exact measurement ureteral length as most existing studies can cite as published by Chew et al., 2007 (3).

Remember important study in our country's existing anatomy of the ureteropelvic junction by Sampaio published in 1996 with great applicability to endopielotomia technique (4).

\section{REFERENCES}

1. Benjamin JA, Tobin CE: Abnormalities of the kidneys, ureters, and perinephric fascia: anatomic and clinical study. J Urol. 1951; 65: 715-33.

2. Wexler NS, Roberts JA: A correlation of anatomic and physiologic parameters in the ureter. Invest Urol. 1974; 11: 286-7.
3. Chew BH, Knudsen BE, Nott L, Pautler SE, Razvi H, Amann $\mathrm{J}$, et al:: Pilot study of ureteral movement in stented patients: first step in understanding dynamic ureteral anatomy to improve stent comfort. J Endourol. 2007; 21: 1069-75.

4. Sampaio FJ: The dilemma of the crossing vessel at the ureteropelvic junction: precise anatomic study. J Endourol. 1996; 10: 411-5.
Dr. Maurício Hachul Urologist Rua Vergueiro 1855 / 114, Vila Mariana Sao Paulo, SP, 04101-000, Brazil

Telephone: + 5511 5579-0008

E-mail: hachul.mauricio@uol.com.br 